\title{
SUBSTRATES FOR THIN CRYSTALLINE SILICON SOLAR CELLS
}

\author{
Andrew W. Blakers \\ Engineering Department \\ Australian National University \\ Canberra, 0200, Australia \\ ph 6162495905 \\ fax 6162490506 \\ email: Andrew.Blakers@anu.edu.au \\ 12th May 1997
}

\begin{abstract}
Choice of substrate for thin crystalline silicon solar cells requires a compromise between cost and quality. There are three generic substrate types, namely a transparent substrate (such as glass), an opaque substrate (such as a ceramic or metal) and low cost multicrystalline silicon. Glass has the advantage of eliminating absorption within the substrate. However, the larger effective diffusion length, the improved surface passivation and the increased process flexibility obtainable with an opaque substrate, particularly low cost multicrystalline silicon, may considerably outweigh the modest optical benefits of a transparent substrate. In this paper it is shown that the advantage in effective diffusion length that is required of a cell grown on an opaque substrate in order to offset the light trapping advantages of a glass substrate is about a factor of two.
\end{abstract}

\section{INTRODUCTION}

Thin crystalline silicon solar cells have considerable promise for photovoltaic applications. They share the benefits of reduced material consumption with other thin film candidates such as amorphous silicon, copper indium diselenide and cadmium telluride but retain the advantages of crystalline silicon. These advantages include high efficiency potential, performance stability, silicon abundance, silicon non-toxicity, the ability to share research and infrastructure costs with the integrated circuit industry and market dominance.

Thin c-Si cell fabrication requires the deposition of a layer of silicon onto a suitable low cost substrate in a low cost manner. The main purpose of the substrate is to provide mechanical support. The substrate is generally electronically inactive. The layer of silicon can be deposited as a large grained crystal directly (for example by epitaxial growth onto a multicrystalline silicon substrate) or can be crystallized after deposition. The choice of substrate strongly influences the method of achieving suitably large grain sizes.

In addition to cost, the criteria to be used to choose a suitable substrate to support c-Si layers include:

- Epitaxial template: Good photovoltaic performance depends on having grains whose lateral dimensions are large (> 100 microns) and greater than their thickness. This is easy to achieve 
if silicon is grown epitaxially on a low cost c-Si substrate with a pre-existing grain structure. No low cost non-silicon material is available for epitaxial growth of silicon.

- High quality grown layer: A substrate that can tolerate a high silicon growth temperature will allow the fabrication of better and larger silicon crystals. However, diffusion of impurities from the substrate into the silicon has to be avoided, through prior cleaning steps or the use of a diffusion barrier.

- Optical Transparency: A transparent substrate avoids loss of long wavelength, weakly absorbed light into the electronically inactive substrate. It also allows the use of light trapping schemes that rely on diffuse reflection from the rear surface of the substrate. Glass is the most likely transparent substrate. An alternative method of improving light trapping is to interpose a dielectric layer between the grown silicon film and the substrate.

- Thermal Expansion Coefficient Matching: Elevated process temperatures can lead to cracking of the c-Si film after cooling if there is a thermal expansion mismatch with the substrate. Low cost c-Si substrates avoid this problem. It is also possible to fabricate ceramics and glasses that are reasonably well matched to silicon. However, design freedom is reduced and costs are increased.

- Electrically insulating: Several cell interconnection schemes rely on the substrate being electrically insulating. Any substrate can have this property, either because the substrate itself is insulating or by pre-deposition of an insulating dielectric.

- Thermally stable: Silicon, ceramics and some metals allow high temperature processing. Some glass types are also stable at elevated temperatures (above $800 \mathrm{deg}$ ). This is an important property since many standard silicon processing steps require high temperatures.

- Low Surface Recombination Velocity: In principle, passivation of the outer surface of a silicon film can be achieved by techniques such as oxidation or nitride deposition (provided that the substrate can tolerate the temperatures required). Passivation of the interface between the silicon and the substrate is more difficult. In many crystallisation techniques crystal growth commences at the interface, and the defect density is therefore likely to be highest in that region. Epitaxial growth on a heavily doped silicon substrate avoids the problem because epitaxy allows a low interface defect density.

The criteria listed above can be used to reduce the range of possible cell designs to three generic types:
A. glass
B. a dielectric coated opaque substrate such as metal, ceramic or low cost multicrystalline silicon
C. an opaque substrate such as low cost multicrystalline silicon

Both type A and type B designs have the advantages that light trapping is assisted by the glass or dielectric layer and the substrate is electrically insulating. However, the quality of c-Si grown in such designs is likely to be inferior to c-Si grown directly onto crystalline silicon in each of the other aspects mentioned above.

The purpose of this paper is to determine the advantage in material quality that is required of a cell grown on a c-Si substrate (design $\mathrm{C}$ ) in order to offset the light trapping advantages of a foreign substrate (designs A or B above). This is accomplished by modelling in a rather general 
way the relative merits of various substrates for thin crystalline silicon (c-Si) solar cells. The analysis is performed by bracketing the likely values of key cell parameters and thereby determining the maximum advantage that a particular substrate can have over alternatives.

\section{MODELING}

The one dimensional solar cell modeling program PC1D [1] was used to calculate cell performance for a range of key cell parameters that will depend on substrate type. The five parameters listed below determine in a rather general way the important generation and recombination processes within solar cells. Other parameters are of secondary importance. The values chosen for each of the parameters bracket the likely values to be found in a commercial solar cell.

- minority carrier diffusion length in the bulk region of the cell $\left(\mathrm{L}_{\underline{n}}\right): 5,10,20,40,80$ or 160 microns

- recombination in the front surface region $\left(\mathrm{S}_{\mathrm{f}}\right): 10^{2}$ or $10^{5} \mathrm{~cm} / \mathrm{sec}$

- recombination in the rear surface region $\left(\mathrm{S}_{\mathrm{r}}\right): 10^{2}$ or $10^{5} \mathrm{~cm} / \mathrm{sec}$

- light trapping quality: textured-Lambertian (excellent) or polished (poor)

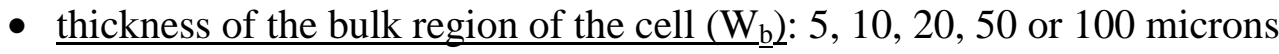

PC1D was run 540 times, spanning the parameter space of the three cell designs (A, B and C) together with the six diffusion lengths, three surface recombination velocity combinations, the five cell thicknesses and the two qualities of light trapping. The other parameters chosen for the study were as follows:

- Cell base resistivity: 1 ohm-cm $\left(\mathrm{n}^{+} / \mathrm{p} / \mathrm{p}^{+}\right)$.

- Front surface diffusion: erfc profile, 0.4 microns deep, maximum doping of $10^{19} \mathrm{~cm}-3$

- Rear surface diffusion: 1 micron thick $\mathrm{p}^{+}$silicon doped at $5 \times 10^{18} \mathrm{~cm}^{-3}$ with a minority carrier diffusion length of 5 microns

- Substrate type in cell Design C: 150 micron thick $\mathrm{p}^{+}$silicon doped at $5 \times 10^{18} \mathrm{~cm}^{-3}$ with a minority carrier diffusion length of 5 microns

- Front surface reflectivity: nil

- Rear surface reflectivity: 95\% for Designs A and C and 40\% for Design B (which represents the reflectivity that could be achieved by insertion of a low refractive index dielectric layer between the opaque substrate and the grown silicon layer)

- Material Properties: as for single crystal silicon

- Excitation: AM1.5G, $100 \mathrm{~mW} / \mathrm{cm}^{2}, 25 \mathrm{deg}$

Minority carrier diffusion lengths of 5 and 160 microns correspond to minority carrier lifetimes of $8 \mathrm{nsec}$ and 8 microsec respectively. They allow upper bound cell efficiencies of $13 \%$ and $22 \%$ respectively (for idealized choices of other parameters). These efficiencies approximate the lower and upper limits of commercially interesting efficiencies respectively, and so the diffusion length range chosen spans the range of economic interest. 
A thin heavily doped layer was assumed to be present at each surface of the cell, which suppressed the surface recombination rate. Three surface recombination velocity combinations were modeled: $\mathrm{S}_{\mathrm{f}}=\mathrm{S}_{\mathrm{r}}=100 \mathrm{~cm} / \mathrm{sec}, \mathrm{S}_{\mathrm{f}}=100 \mathrm{~cm} / \mathrm{sec} \& \mathrm{~S}_{\mathrm{r}}=10^{5} \mathrm{~cm} / \mathrm{sec}$ and $\mathrm{S}_{\mathrm{f}}=\mathrm{S}_{\mathrm{r}}=10^{5} \mathrm{~cm} / \mathrm{sec}$. These parameters imposed $\mathrm{V}_{\text {oc }}$ limits (for large diffusion lengths) of $683 \mathrm{mV}, 651 \mathrm{mV}$ and $623 \mathrm{mV}$ respectively. The $\mathrm{V}_{\mathrm{oc}}$ limits represent extremes in the likely values to be found in a commercial cell.

A textured surface (Lambertian light trapping scheme) and a polished surface are respectively better and worse than the light trapping scheme that would be found in any commercial solar cell. The encapsulated V-texture design [2] is therefore covered by this analysis.

The silicon thickness was optimised for all of the combinations of parameters listed above. The optimum thickness is larger for polished than for textured cells and for Design C than for Design A cells because of less effective light trapping. The optimum base thickness lay in the range 5 to 100 microns in every case. The optimum base thickness was generally $50-100 \%$ of the diffusion length.

Although only one resistivity was examined, the range of diffusion lengths chosen was sufficiently large to span the likely range of diffusion length - doping density products. The material properties were chosen to be those of single crystal silicon. Provided that the defect density is not very high within grains this is a reasonable assumption. Grain boundaries, of course, can cause some of the bulk material properties such as mobility to differ substantially from those of single crystal silicon. In small grained silicon the effective diffusion length will be determined by the grain size and not by the intrinsic material quality.

Proper consideration of grain boundaries requires a two or three dimensional modelling program. However, a one dimensional model is sufficient for the purposes of comparing idealised small grained silicon deposited on a foreign substrate with larger grained silicon grown by epitaxy on a silicon substrate because nearly all two and three dimensional processes reduce rather than increase cell performance. The use of a one dimensional model favours Designs A and B since the grain sizes will be considerably smaller than in Design C.

A conventional cell structure (single junction) was chosen for the modelling. It has been suggested that multiple junctions can enhance cell performance for poor quality silicon [3]. However, comprehensive modelling has shown that the efficiency advantage arising from an increased current from such a solar cell is cancelled by reductions in voltage and fill factor [2,4]. Potential disadvantages of a multiple junction design include increased junction shunting in small grained silicon and problems with device fabrication if silicon deposition and crystallisation involves silicon melting.

\section{RESULTS}

The cell thickness, which can be freely chosen by the cell designer in most circumstances, was optimised to yield maximum efficiency. Eighteen data sets were then generated of efficiency as a function of diffusion length, comprising combinations of the three cell designs (A, B and C), the 
two light trapping schemes and the three surface recombination velocity combinations. The data sets were curve-fitted to allow interpolation. Six of the data sets are shown in figure 1.

As expected, the light trapping properties of a glass substrate yield an advantage over an opaque substrate for similar cell diffusion lengths and surface recombination velocities. The additional diffusion length required of a cell fabricated on an opaque substrate to overcome this advantage can be determined. The additional diffusion length required is itself weakly dependent on material quality. Table 1 lists the ratio of diffusion length required for equal cell efficiencies averaged over the diffusion length range 5 to 160 microns. The standard deviation of the average is included to illustrate that the ratio is only a weak function of diffusion length.

Table 1 shows that the diffusion length of a cell of design B or C should be $50-100 \%$ larger than that of a cell of Design A for equal efficiency potential.

Table 1: Average ratio of diffusion lengths $\left(\mathrm{L}_{B} / \mathrm{L}_{A}\right.$ or $\left.\mathrm{L}_{C} / \mathrm{L}_{\mathrm{A}}\right)$ required to match efficiencies of cells of Designs B and C with cells of Design A. The standard deviation of the average is included.

\begin{tabular}{|l|c|c|c|c|c|c|}
\hline Surface finish & textured & textured & textured & polished & polished & polished \\
\hline $\mathrm{S}_{\mathrm{f}}(\mathrm{cm} / \mathrm{sec})$ & 100 & 100 & $10^{5}$ & 100 & 100 & $10^{5}$ \\
\hline $\mathrm{S}_{\mathrm{r}}(\mathrm{cm} / \mathrm{sec})$ & 100 & $10^{5}$ & $10^{5}$ & 100 & $10^{5}$ & $10^{5}$ \\
\hline ratio $\mathrm{L}_{\mathrm{B}} / \mathrm{L}_{\mathrm{A}}$ & 2.15 & 2.02 & 2.12 & 1.38 & 1.34 & 1.36 \\
(standard deviation) & $(18 \%)$ & $(22 \%)$ & $(26 \%)$ & $(10 \%)$ & $(15 \%)$ & $(15 \%)$ \\
\hline ratio $\mathrm{L}_{\mathrm{C}} / \mathrm{L}_{\mathrm{A}}$ & 2.74 & 2.20 & 2.33 & 1.53 & 1.31 & 1.34 \\
(standard deviation) & $(19 \%)$ & $(27 \%)$ & $(30 \%)$ & $(8 \%)$ & $(15 \%)$ & $(15 \%)$ \\
\hline
\end{tabular}

Figure 2 graphs the ratio of the efficiency of cells of designs $\mathrm{B}$ and $\mathrm{C}$ to that of design $\mathrm{A}$ as a function of the ratio of the base diffusion length of cell designs $\mathrm{B}$ and $\mathrm{C}$ to that of design $\mathrm{A}$. Twelve curves are shown on the graph, corresponding to the 12 possible combinations of the two light trapping qualities, the three surface recombination velocities and the two designs B and C. The curves divide into two groups: polished and textured. Within each group the curves lie on top of each other. The exception to this is Design $\mathrm{C}$ cells with a surface recombination velocity of $100 \mathrm{~cm} / \mathrm{sec}$ at each surface. In the case of excellent surface passivation Design $\mathrm{C}$ cells require a larger diffusion length advantage in order to match the efficiency of Design A cells. As expected, the advantage of a glass substrate is less for a polished cell.

A low recombination velocity at the rear surface is necessary to obtain the full benefits of a transparent substrate. Otherwise the optimum base thickness for Designs A and B (but not Design C) shifts to larger values in order to minimise rear surface recombination. Obtaining a low surface recombination at the interface between silicon and a foreign substrate will be difficult.

\section{$\underline{\text { DISCUSSION }}$}

It is likely that growth of crystalline silicon on glass will result in substantially lower cell effective diffusion lengths than growth on a substrate chosen specifically to yield good quality 
silicon. Such a substrate would have a similar thermal expansion coefficient to that of silicon and would tolerate high temperatures, allowing a free choice of growth (and cell processing) conditions. In the case of a low cost multicrystalline silicon substrate (Design C) an epitaxial template would also be present.

Deposition of silicon on a foreign substrate at a low temperature typically yields silicon with grain sizes below 10 microns. The effective diffusion length in such material would be of the order of the grain size. Potentially serious problems include junction shunting along grain boundaries, and an inability simultaneously to achieve tolerable grain size (eg by melt recrystallisation) and to tailor doping profiles in the device in order to maximise cell performance. Recrystallization of a deposited layer by laser or electron beam spot heating generally involves extremely rapid cooling, leading to high defect densities. Restrictions on processing temperature eliminates standard cell processing techniques such as diffusions and oxidations and it will be necessary to develop affordable alternatives, including the associated processing equipment.

Figure 2 shows that a cell with a 100 micron diffusion length fabricated on an opaque substrate would have approximately a 30\% (relative) potential efficiency advantage over a cell fabricated on glass with 10 micron grain size. Minority carrier diffusion lengths in excess of 150 microns are readily obtained by liquid phase epitaxial growth of silicon onto multicrystalline silicon substrates [5,6]. Large grained silicon can also be obtained by techniques such as strip heater recrystallisation of deposited silicon. Both of these techniques are performed at temperatures above 900 degrees, which is out of the temperature range of all but specialised glass.

An advantage of multicrystalline silicon as a substrate is that the recombination velocity at the interface between the grown silicon and the substrate is likely to be much lower than at the interface between silicon and a foreign substrate..

\section{CONCLUSION}

The benefits of a transparent substrate such as glass over opaque alternatives for thin crystalline solar cells are rather small, and are unlikely to exceed 15\% (relative) even for favourable values of cell parameters. The disadvantages of a glass substrate include restrictions on process temperatures, thermal expansion mismatch, difficulties with passivation of the silicon surface lying at the interface with the substrate and small grain size. The optical advantage of glass relative to an opaque substrate is neutralised if the silicon grown on the opaque substrate has an effective diffusion length more than twice that of the silicon grown on glass. It is likely that the larger effective diffusion length, the improved surface passivation and the increased process flexibility obtainable with an opaque substrate will outweigh the modest optical benefits of a glass substrate. In particular, low cost, low quality multicrystalline silicon is a promising substrate for thin crystalline silicon solar cells despite the loss of some long wavelength light into the substrate. 


\section{REFERENCES}

1 PC1D v4.4, P. Basore, University of NSW, 1996

2 U. Rau, T. Meyer, M. Goldbach, R. Brendel and J.H. Werner, "Numerical Simulation of Innovative Device Structures for Silicon Thin Film Solar Cells", Proc. $25^{\text {th }}$ IEEE Photovoltaic Spec. Conf., pp 469-472, Washington, 1996

3 M.A. Green and S.R. Wenham, "Novel Parallel Multijunction Solar Cell", Appl. Phys. Lett., Vol 65, pp 2907-2909 (1994)

4 M. Stocks, A. Cuevas and A.W. Blakers, "Theoretical Comparisons of Conventional and Multilayer Thin Silicon Solar Cells", Progress In Photovoltaics, Vol 4, pp 35-54, 1996

5 A.W. Blakers and K.J. Weber, Multicrystalline Silicon Solar Cells by LPE", Proc. $6^{\text {th }}$ Workshop on the Role of Defects and Impurities in Silicon Device processing, pp 64-73, Snowmass, Colorado, 1996

6 J.H. Werner, J.K. Arch, R. Brendel, G. Langguth, M. Konuma and E. Bauser, "Crystalline Thin Film Silicon Solar Cells", Proc. $12^{\text {th }}$ EV Photovoltaic Solar Energy Conference, Amsterdam, 1994

\section{ACKNOWLEDGEMENTS}

The Australian Research Council provided support for this work.

\section{FIGURE CAPTIONS}

Figure 1: Cell efficiency (optimised for base thickness) as a function of base diffusion length for cell Designs A, B and C. Front and rear surface recombination velocities are $10^{2}$ and $10^{5} \mathrm{~cm} / \mathrm{sec}$ respectively. A Lambertian light trapping scheme is assumed in figure 1a while a polished surface is assumed in figure $1 \mathrm{~b}$.

Figure 2: Ratio of efficiency of cell designs $\mathrm{B}$ and $\mathrm{C}$ to that of design $\mathrm{A}$ as a function of the ratio of the base diffusion length of cell designs $\mathrm{B}$ and $\mathrm{C}$ to that of design A. See text for details. 